\section{HSE}

Historia Social y de la Educación

Social and Education History
Hipatia Press

www.hipatiapress.com

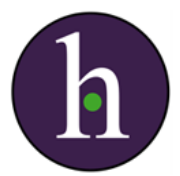

Instructions for authors, subscriptions and further details:

http://hse.hipatiapress.com

\title{
Memorias Adaptables para la Construcción de Identidades Colectivas
}

\section{Lucas Bietti ${ }^{1}$}

1) University of Neuchâtel, Switzerland

Date of publication: June $23^{\text {th }}, 2018$

Edition period: June 2018-October 2018

To cite this article: Bietti, L. (2018). Memorias Adaptables para la Construcción de Identidades. Social and Education History, 7(2), 125-150. doi:10.17583/hse.2018.2820

To link this article: http://dx.doi.org/10.17583/hse.2018.2820

\section{PLEASE SCROLL DOWN FOR ARTICLE}

The terms and conditions of use are related to the Open Journal System and to Creative Commons Attribution License (CC-BY). 


\title{
Memorias Adaptables para la Construcción de Identidades Colectivas
}

\author{
Lucas Bietti \\ University of Neuchatel (Switzerland)
}

\section{Resumen}

La investigación sobre la maleabilidad de la memoria humana ha mostrado los diferentes modos en los que podemos incorporar información errónea dadas ciertas condiciones sociales. Esta línea de investigación ha llevado a psicólogos cognitivos a centrarse en los errores de la memoria y la desinformación que pueden ser causados por el contagio social. Estudios recientes han comenzado a analizar los posibles beneficios que conllevan el contagio social de la memoria. Dicha línea de investigación se ha centrado en las características potencialmente adaptables de la memoria. Estos estudios sugieren que la adaptabilidad de la memoria ayuda a promover la cooperación en grupos sociales y desempeña un papel central en el desarrollo de la confianza entre miembros de grupos sociales. La adaptabilidad de la memoria crea las condiciones necesarias para la formación de comunidades de memoria, que son la base para la construcción y la transmisión de memorias colectivas. La memoria colectiva puede ser definida como memorias individuales compartidas en una comunidad, que le confieren identidad a la comunidad. Las memorias colectivas pueden pertenecer a un proyecto identitario que los miembros de grupos sociales emplean para conservar su historia y mantener cohesión. El objetivo de nuestro artículo es investigar cuáles son los factores sociales y cognitivos que promueven la formación de memorias colectivas en interacciones sociales. Para esto discutimos numerosos estudios en psicología social, cultural y cognitiva que se han centrado en explicar los beneficios y costos de formar memorias colectivas. La evidencia empírica sugiere que la adaptabilidad de la memoria humana, y en consecuencia la capacidad que grupos sociales tienen para formar memorias colectivas sobre las cuales basar sus identidades sociales, parece ser bastante independiente de la veracidad y precisión de tales memorias.

Palabras clave: Memoria, adaptabilidad, evolución, artefactos, identidad 


\section{Adaptive Memories for the Construction of Collective Identities}

Lucas Bietti

University of Neuchâtel (Switzerland)

\section{Abstract}

Research on the malleability of human memory has shown how we can incorporate misinformation under certain social and cognitive conditions. This line of inquiry has led cognitive psychologists to focus on memory errors and misinformation effects caused by social contagion. Based on the same social contagion effect, recent studies have focused on its potentially adaptive features, as a trust-based mode for promoting cultural learning and cooperation among in-group members and enabling the formation and maintenance of mnemonic communities, which are the basis for the emergence, formation and transmission of collective memories. We may consider that collective memories can be operationalized as individual memories shared across a community that bear on the community's identity. These collective memories may belong to an identity project that members of groups often use to preserve an established group history and maintain group cohesion. The aim of this article is to investigate which are the social and cognitive resources that promote the formation of collective memories in social interactions. In order to do so, we discuss several studies in social, cultural and cognitive psychology that have focused on studying the benefits and costs of having collective memories. The empirical evidence suggests that the adaptability of human memory, and therefore, the ability of social groups to create collective memories upon which to base their collective identities, seems to be quite independent of the veracity and accuracy of such memories.

Keywords: Memory, adaptability, evolution, identity 


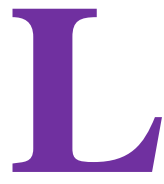

a memoria colaborativa implica situaciones en las que dos o más personas recuerdan de manera conjunta experiencias pasadas que pueden haber sido compartidas (Mario y Liliana recuerdan sus últimas vacaciones en París). También podemos recordar conjuntamente experiencias similares (ej. visitar el Centro GeorgePompidou) que atravesamos de manera independiente (ej. Mario lo había visitado años antes en su primera visita a París mientras que Liliana lo visitó por primera vez en sus últimas vacaciones con Mario). En consecuencia, la información que Liliana y Mario recuerdan acerca de su visita al Centro George-Pompidou de forma colaborativa puede ser el resultado de haber codificado una misma o similar experiencia de forma compartida o separada (Barber, Rajaram, \& Aron, 2010; Barnier, Sutton, Harris, \& Wilson, 2008; Harris, Barnier, \& Sutton, 2013; Pereira-Pasarin \& Rajaram, 2011). Por supuesto que nuestras memorias individuales y colaborativas no siempre representan de forma acertada los acontecimientos que ocurrieron en el pasado, más bien funcionan como representaciones individuales y compartidas de ese pasado (Sutton, 2008). Tal re-evocación de experiencias individuales y/o compartidas se basa en la capacidad humana de poder viajar mentalmente en el tiempo: "esta facultad permite a los seres humanos proyectarse mentalmente en el pasado para revivir etapas de la vida o hacia el futuro para anticipar posibles eventos y sus consecuencias" (Suddendorf \& Corballis, 2007, p. 299).

Numerosos estudios se han dedicado a medir los beneficios y costos de la memoria colaborativa (ej. Meade et al., 2009; Weldon \& Bellinger, 1997). Estas investigaciones han demostrado que el recuerdo colaborativo puede tanto inhibir o facilitar la capacidad individual de recordar tales experiencias (ej. Rajaram, 2011). Las actividades colaborativas (ej. conversaciones) en las que recordamos hechos compartidos se basan en continuas interacciones entre recursos cognitivos internos (nuestra memoria a nivel biológico) y recursos cognitivos externos (como otras personas y la tecnología) (Donald, 1993; Michaelian \& Sutton, 2013; Sterelny, 2012; Sutton et al., 2010). Estas constantes y repetidas interacciones conducen a una acumulación de conocimientos y habilidades a través del tiempo. Tal acumulación de conocimientos y habilidades transforma los contextos sociales y culturales 


\section{Bietti - Construcción de identidades colectivas}

en los que nuevas generaciones habitan, afectando de ese modo, su desarrollo ontogenético (Sterelny, 2012). La transmisión de conocimientos y habilidades a lo largo del tiempo permite la formación de memorias colectivas (Wertsch, 2002, 2009) dentro de comunidades mnemotécnicas específicas (Zerubavel, 2003).

El objetivo de nuestro artículo es investigar cuáles son los factores sociales y cognitivos que promueven la formación de memorias colectivas en interacciones sociales. Para esto discutimos numerosos estudios experimentales en psicología social, cultural y cognitiva que se han centrado en explicar los beneficios y costos de formar memorias colectivas. La evidencia empírica sugiere que la adaptabilidad de la memoria humana, y en consecuencia la capacidad que grupos sociales tienen para formar memorias colectivas sobre las cuales basar sus identidades sociales, parece ser bastante independiente de la veracidad y precisión de tales recuerdos.

En la primera parte del artículo analizamos estudios experimentales en psicología cognitiva que han demostrado que recordar de manera colaborativa (ej. conversaciones) en grupos sociales tiende más a afectar que a facilitar la capacidad individual de recordar. Es decir, estos estudios observaron que solemos recordar más información con relación a una experiencia pasada cuando lo hacemos de manera individual que cuando lo realizamos en grupo. En otras palabras, rara vez recordar experiencias pasadas con otras personas nos lleva a recordar más información en comparación de cuando lo hacemos de forma individual.

En la segunda parte discutimos numerosos trabajos que han analizado cómo las memorias son transmitidas a través de cadenas complejas de comunicación a lo largo tiempo. Del mismo modo, examinamos los costos que estas prácticas conllevan y los efectos del contagio social para la memoria. En la tercera parte sostenemos que los costos para la memoria individual relacionados a recordar de manera colaborativa en interacciones sociales están determinados, en gran medida, por la maleabilidad y la adaptabilidad de la memoria humana. En la cuarta parte indicamos que la maleabilidad y la adaptabilidad de la memoria humana, en continua interacción con recursos cognitivos externos, han permitido el surgimiento de redes híbridas, que incorporan aspectos culturales y cognitivos a través de nuestra historia cultural y evolutiva (Donald, 1991). Estas redes de naturaleza híbrida contienen elementos culturales y cognitivos creando las 
condiciones para el surgimiento de memorias colectivas a gran escala. En la última parte del artículo discutimos las razones por las que contar con memorias adaptables permite la construcción de identidades colectivas.

\section{La Memoria en Grupos Pequeños}

Hablar de un pasado compartido en compañía de otras personas es uno de los modos más frecuentes para construir memorias colectivas (Bietti, 2012, 2014; Middleton \& Brown, 2005; Hirst \& Echterhoff, 2012). En estas conversaciones acerca de un pasado compartido solemos coordinar recursos lingüísticos (ej. estructuras sintácticas) y corporales (ej. gestos, miradas, postura corporal y expresiones faciales) con el fin de lograr objetivos comunes (ej. tratar de recordar conjuntamente dónde dejamos el auto estacionado). Tales conversaciones son actividades colaborativas (Clark, 1996; Clark \& Brennan, 1991) en las que los participantes tienen que "compartir y sincronizar aspectos de sus estados mentales para actuar juntos en el mundo" (Brennan, Galati \& Kuhlen, 2010, p 304).

Coordinar aspectos cognitivos, lingüísticos y corporales con las personas con las que recordamos un pasado compartido de forma colaborativa en conversaciones suele ocurrir con poco esfuerzo y de modo natural (Cienki, Bietti \& Kok, 2014). Sin embargo, recordar con otras personas no sólo tiene que ver con la coordinación de estos recursos a través del tiempo. Los participantes, en algún momento de la actividad colaborativa, tienen que crear una versión compartida del pasado, a diferencia de lo que ocurre mientras interactuamos para resolver un problema, imaginarnos situaciones contrafácticas o proyectarnos hacia el futuro (Bietti \& Sutton, 2015). En el proceso de crear una versión compartida del pasado factores como el estilo de comunicación (Harris et al., 2011; Meade et al., 2009), grado de familiaridad de los participantes (Harris, Barnier \& Sutton, 2012; Rajaram \& Pereira-Pasarin, 2010) así como el hecho de haber codificado esa experiencia de forma conjunta (Harris et al., 2013) tienen un papel central en qué y cómo recordamos en interacción con otras personas.

En la próxima sección presentamos el concepto de memoria transactiva (Wegner, 1986) y nos centramos en discutir los resultados obtenidos de 


\section{Bietti - Construcción de identidades colectivas}

manera experimental dentro del campo de la psicología cognitiva (Rajaram, 2011; Weldon \& Bellinger, 1997).

\section{La Memoria Transactiva}

Numerosos estudios en psicología organizacional, psicología del aprendizaje, psicología cognitiva y filosofía de la mente mostraron que equipos de expertos, grupos de estudiantes y parejas que han pasado mucho tiempo juntos pueden formar sistemas de memorias transactivas (Hollingshead, 1998; Jackson \& Moreland 2009; Lewis, Lange, \& Gillis, 2005; Ren \& Argote, 2011; Sparrow, Liu, \& Wegner, 2011; Theiner, 2013; Wegner, 1986). Un sistema de memoria transactiva se rige por "el funcionamiento de los sistemas de memoria de las personas y los procesos de comunicación que se producen dentro del grupo" (Wegner, 1986, p. 191). La composición y el desarrollo de un sistema de memoria transactiva necesita que: i) la información a recordar se encuentre distribuida entre los integrantes del grupo; y ii) que cada miembro del grupo sepa que otro de los miembros posee información específica que el primero no posee y que puede contar con su colaboración cuando sea necesario. Si estos dos requisitos se cumplen, las personas dentro de un sistema de memoria transactiva cuentan con la posibilidad de distribuir su trabajo cognitivo de tal manera que cada miembro sólo asume la responsabilidad de aprender y recordar la información dentro de su propio dominio de conocimiento - sabiendo (explícita o implícitamente) que otro de los miembros del sistema de memoria transactiva es responsable de otra área de especialización. De este modo, las personas que forman parte de un sistema de memoria transactiva no desperdician recursos cognitivos intentando recordar información que otro miembro del sistema debe recordar y es experto. Esto lleva a que los grupos que son capaces de desarrollar sistemas de memoria transactiva se encuentren en mejores condiciones en tareas de memoria, en comparación de aquellos en los que esta distribución del trabajo cognitivo no sucede (Wegner, Erber, \& Raymond, 1991). 


\section{Beneficios y Costes de la Colaboración}

La investigación en memoria transactiva en psicología organizacional, por ejemplo, ha tendido a satisfacerse con el criterio según el cual el grupo inevitablemente supera al individuo en el momento de recordar información (Ren \& Argote, 2011). De este modo, si un grupo supera a uno de sus miembros en tareas de memoria, se entiende que esto ocurrió por la capacidad del grupo de desarrollar y poner en funcionamiento un sistema de memoria transactiva.

Sin embargo, en psicología cognitiva, los estudios experimentales que han analizado los modos en los que miembros de grupos recuerdan en forma colaborativa no se han satisfecho con el anterior criterio. En psicología cognitiva el rendimiento del grupo no sólo debe ser superior al de uno de sus miembros, sino también a la suma de los rendimientos de cada uno de sus miembros recordando de manera individual. Estos estudios encontraron que recordar en grupo frecuentemente perjudica el rendimiento individual de cada uno de sus miembros (Basden et al., 1997; Rajaram \& Pereira-Pasarin, 2010; Weldon \& Bellinger, 1997). En otras palabras, recordar en grupo, en muchas ocasiones, inhibe más que mejora la capacidad de recordar a nivel individual. Basden y colaboradores han explicado este fenómeno por medio de "la hipótesis de la interrupción en la recuperación de información" (Basden, Basden \& Henry, 2001). Esta hipótesis sostiene que escuchar las respuestas de otras personas altera la forma en que cada individuo organiza su secuencia de recuerdo y estrategias de recuperación de la información. Esto le impide a cada miembro alcanzar su más alto potencial cuando recuerda en grupo (Barber et al., 2010; Barber \& Rajaram, 2011; Basden et al., 1997; Rajaram, 2011).

No obstante, dentro de esta línea de investigación estudios recientes sugieren que recordar con otras personas puede ser al menos no perjudicial para la memoria individual y hasta incluso beneficioso, en ciertos grupos y bajo ciertas circunstancias (Harris et al., 2011; Meade et al., 2009). Por ejemplo, en un estudio que comparó el rendimiento en tareas de memoria colaborativa en grupos de pilotos expertos y no expertos, se encontraron beneficios en los primeros (Meade et al., 2009). Es decir, el rendimiento en los grupos de pilotos expertos fue mejor que la suma de los rendimientos de cada uno de sus miembros recordando por separado. Dicho efecto no se 


\section{Bietti - Construcción de identidades colectivas}

encontró en los grupos de no expertos donde se observó inhibición de la memoria individual como resultado de la colaboración. Meade y sus colegas analizaron las conversaciones en ambos tipos de grupos (pilotos expertos y no expertos), y descubrieron que en los grupos de expertos uno de los factores clave para tener mejor rendimiento fue el hecho de contar con estrategias comunicativas más elaboradas. Un ejemplo de las tales estrategias era el constante y recíproco monitoreo de la comprensión por medio de repeticiones y reformulaciones que actuaban para explicitar el conocimiento compartido. Estas estrategias comunicativas estuvieron ausentes en los grupos de no expertos que mostraron inhibición de la memoria individual como consecuencia de la colaboración. Meade y sus colegas sostienen que las estrategias comunicativas encontradas en los grupos de expertos fueron el resultado de su formación y experiencia profesional, en la que el intercambio de información es crucial.

En otra serie estudios focalizados en los beneficios y costos de la colaboración en tareas de memoria, Harris y sus colegas (2011) encontraron que, bajo ciertas condiciones, parejas mayores (que han compartido gran parte de su vida) pudieron recordar información que ambos individuos parecieron haber olvidado si intentaban recordarla de forma individual. No obstante, las condiciones y la clase de material que se les pidió a las parejas recordar de modo conjunto e individual desempeñó un papel central facilitando o inhibiendo su rendimiento. Los factores que llevaron a tales beneficios incluyeron estrategias comunicativas para recuperar información y repeticiones para establecer acuerdos y hacer explícito el conocimiento compartido (Harris et al., 2011). Es decir, los resultados obtenidos por Harris y sus colegas estuvieron en concordancia con los reportados en el estudio de pilotos expertos y no expertos (Meade et al., 2009). A saber, en ambos estudios, el estilo de la interacción verbal y la comunicación jugaron un papel central en la mejora del rendimiento en tareas de memoria.

Sumado al papel de las estrategias comunicativas en la aparición de efectos de facilitación en tareas de memoria colaborativa, Harris y sus colegas (2013) examinaron si las condiciones (individuales vs. compartidas) en las que los participantes codificaron la información para luego ser recordada también influyó en su posterior rendimiento. Los resultados de dicho estudio mostraron una correlación positiva entre el hecho de haber codificado de manera conjunta la información para luego 
ser recordada y la eliminación de fenómenos de inhibición en la memoria individual en las tareas colaborativas (Harris et al., 2013). Estos resultados fueron encontrados en grupos de participantes que se conocían anteriormente al estudio como así también en desconocidos. Tales resultados sugieren que las estrategias comunicativas y el hecho de haber codificado de forma conjunta la información se relacionan con una colaboración efectiva cuando los participantes de ambos estudios tuvieron que recordar en grupo.

\section{La Transmisión Social de la Memoria}

En esta sección discutimos cómo se transmiten las memorias a lo largo de cadenas de comunicación en grupos sociales. Luego nos centramos en explorar los diferentes modos en que distintos factores sociales pueden contaminar estas memorias con información falsa. Esto ocurre por su alta capacidad de adaptación.

\section{Transmisión en Cadenas de Comunicación}

Los estudios del psicólogo Frederic Bartlett (1932) sirven de marco general para entender los modos en que la memoria es susceptible de transformación como resultado de su simple reproducción y transmisión en grupos sociales. Bartlett (1932) empleo dos técnicas para investigar tales fenómenos: i) la reproducción repetida; y ii) la reproducción en serie. En la reproducción repetida los participantes de su estudio debían primero aprender un material nuevo y luego recordarlo en múltiples ocasiones a lo largo del tiempo, sin estar expuestos al material original. Por otro lado, en la reproducción en serie, se le pedía a un participante (A1) que aprendiera un material nuevo (ej. una serie de imágenes e historias) y luego que tuviera que escribir o dibujar de memoria, dependiendo del estimulo original, el material recientemente aprendido. A continuación, A1 debía abandonar la sala donde el experimento se llevaba a cabo, y un nuevo participante (A2) tenía que ver el dibujo o leer la historia generada previamente por A1. Inmediatamente, el experimentador le pedía a A2 que dibujara o escribiera de memoria el nuevo material recientemente aprendido. En los experimentos originales, las cadenas de 


\section{Bietti - Construcción de identidades colectivas}

transmisión se encontraban conformadas por diez participantes (Bartlett, 1932). Al comparar el rendimiento de los participantes en ambas condiciones (reproducción repetida y reproducción en serie), en la reproducción repetida fue cuando más información se había conservado a lo largo del tiempo (Bartlett, 1932; Bergman \& Roediger, 1999; Roediger, Meade \& Bergman, 2001; Roediger, Meade, Gallo \& Olson, 2014). El mejor rendimiento en la reproducción repetida se basó en que reproducir múltiples veces la misma historia o dibujo por un mismo participante funcionó consolidando la memoria del estímulo y mejorando su recuerdo futuro (Roediger et al., 2014). Este fenómeno estuvo ausente en la reproducción en serie, en la que las memorias fueron transmitidas a través de cadenas de comunicación que involucraban múltiples participantes. Esto dio como resultado que la reconstrucción final de la memoria por parte de A10 resultara considerablemente diferente al estímulo originalmente presentado a A1. Semejante variación hacía difícil rastrear las transformaciones que aparecían cada vez que el mismo material era aprendido y reproducido por diferentes participantes. No obstante, si lo que intentamos hacer es entender cómo la transmisión de memorias ocurre a lo largo del tiempo, dentro y a través de grupos sociales y comunidades diversas, nuestro foco de interés no se tendría que centrar tanto en la reproducción repetida, sino más bien en la reproducción en serie. Es decir, para analizar cómo las memorias, los conocimientos y las habilidades se transmiten a través del tiempo no interesaría tanto saber cómo un mismo individuo recuerda una misma historia en múltiples ocasiones, sino como lo hacen múltiples individuos y la forma en cómo dichas memorias se transforman como consecuencia de ello. De ahí que la reproducción en serie sea mas relevante para investigar las dinámicas sociales que llevan a la transmisión y reproducción de memorias a lo largo del tiempo.

\section{La Reproducción en Serie}

El método de reproducción en serie basado en el uso de imágenes e historias como estímulos para luego ser recordados y transmitidos por otros individuos ha sido utilizado en numerosos estudios experimentales que se centraron en analizar los modos en que las memorias cambiaban a lo largo 
del tiempo (Bietti, Mayor \& Bangerter, 2017; Mesoudi \& Whiten, 2008; Roediger et al., 2014; Xu \& Griffiths, 2010). En consonancia con los primeros resultados obtenidos por Bartlett (1932), los nuevos estudios observaron que las memorias de los participantes se modificaban significativamente a medida que más se alejaban del estímulo original y la primera transmisión dentro de la cadena de comunicación. Es decir, se observaron mayores transformaciones del estímulo original a medida que las reproducciones se alejaban de la primera transmisión (ej. Mesoudi \& Whitten, 2004). Entre la clase de transformaciones que fueron encontradas, aparecieron generalizaciones y abstracciones del contenido del estímulo original (Mesoudi \& Whitten, 2004), distorsiones motivadas por sesgos de la memoria, tales como la selección comprensiva y efectos contextuales (Xu \& Griffiths, 2010) y estereotipos culturales (Bangerter, 2000; Kashima, 2000).

Un estudio reciente examinó si la interacción social podía afectar las cadenas de comunicación donde las memorias eran transmitidas (Tan \& Fay, 2011). Tan y Fay (2011) compararon los resultados en términos de cantidad de información preservada a lo largo del tiempo en dos condiciones: i) en cadenas de comunicación en las que los participantes primero tuvieron que escuchar grabaciones de audio de narraciones generadas por una generación anterior de participantes y luego grabar sus propias narraciones de estas historias para que una nueva generación las pudiera escuchar; y ii) en cadenas de comunicación en las que la transmisión involucraba participantes localizados de modo adyacente e interactuando libremente. Los resultados del estudio experimental mostraron que, en la segunda condición, es decir cuando los participantes tuvieron que transmitir sus memorias cara a cara a una nueva generación de participantes, se observaron un menor número de abstracciones y no se encontraron generalizaciones. En otras palabras, más información fue preservada como consecuencia de la interacción social (Tan \& Fay, 2011). Una de las razones que Tan y Fay propusieron para explicar estos resultados fue que el feedback de los oyentes de la nueva generación desempeñó un papel central en el momento de recuperar la información (ej. en términos de exactitud) por parte de la generación anterior. 


\section{Bietti - Construcción de identidades colectivas}

\section{El Contagio Social de las Memorias}

Las investigaciones centradas en examinar la maleabilidad de la memoria humana (Loftus, 1979, 2005) ha mostrado cómo, bajo ciertas condiciones sociales y cognitivas, podemos fácilmente incorporar información errónea en nuestros recuerdos. Esta línea de investigación ha llevado a psicólogos cognitivos y forenses ha estudiar los errores de la memoria causados por el contagio social de los recuerdos (Numbers, Meade \& Perge, 2014; Meade \& Roediger, 2002; Roediger et al., 2001). En varios estudios experimentales se emplearon confederados para insertar información incorrecta en los participantes. En estos estudios se encontró que los participantes recordaron más información incorrecta insertada por confederados que nuevos elementos incorrectos insertados por ellos mismos a causa de sesgos de memoria (ej. Roediger et al., 2001). Otras investigaciones han corroborado estos resultados: las memorias falsas de los confederados insertadas en situaciones interaccionales fueron altamente contagiosas y afectaron negativamente las memorias de los participantes (Echterhoff, Groll, \& Hirst, 2007; Hirst \& Echterhoff, 2012). Recientemente estudios en esta línea de investigación han comenzado a explorar los aspectos positivos de contar con memorias adaptables y fácilmente 'contaminables' (Fagin, Yamashiro \& Hirst, 2013; Wheeler, Allan, Tsivilis, Martin \& Gabbert, 2013). Wheeler y sus colegas sostienen que tener memorias adaptables juega un papel central para la promoción de la confianza entre los miembros de grupos sociales. Esto facilita la transmisión de conocimientos y la cooperación. Tener memorias adaptables y fácilmente 'contaminables' parece ser también de vital importancia en la formación y conservación de comunidades de memoria (Zerubavel, 2003) que son la base para el surgimiento, la formación y la transmisión de la memoria colectiva (Fagin, Yamashiro \& Hirst, 2013).

\section{La Memoria Colectiva}

La formación de la memoria colectiva resulta de mayor complejidad que la transmisión de la memoria en cadenas de comunicación o de los posibles 
efectos causados por el contagio social de los recuerdos. Las memorias colectivas se basan en redes cognitivas distribuidas que incluyen individuos con sus mentes corporizadas en interacción con estructuras institucionales (ej. bibliotecas) y la tecnología (Donald, 1991). Un ejemplo bien conocido de cómo estas redes cognitivas distribuidas operan de sustento para la formación de recuerdos socialmente distribuidos se puede encontrar en los procesos a través de los cuales se enseña y se aprende la historia nacional en las escuelas. Cuando los niños comienzan a aprender la historia de sus países dentro del entorno institucional de la escuela primaria obtienen información proveniente de múltiples fuentes y recursos. Estos incluyen sus recuerdos, libros de texto y rituales institucionales (ej. conmemoraciones de fechas importantes para la historia nacional). Los alumnos de escuela primaria también se hallan expuestos a los recuerdos y las historias de sus padres y familia cercana, así como a la información y valoraciones que puedan venir de productos culturales, tales como la televisión, el cine y el Internet. En el tiempo ontogenético del desarrollo de los niños, las historias nacionales pueden ser sometidas a revisión, y en consecuencia las opiniones de los maestros, así como las versiones presentadas en los libros de texto también pueden cambiar. Los amigos, las propias lecturas e intereses personales también pueden influir moldeando los conocimientos acerca del pasado nacional. Las tecnologías empleadas para transmitir este tipo de historias también lo pueden hacer, desde el Internet y el desarrollo de las redes sociales hasta el uso de la realidad virtual y la tecnología interactiva de tipo tangible. Asimismo, estos elementos formadores de redes cognitivas distribuidas también co-evolucionan con los mismos niños lo largo del tiempo.

En este apartado discutimos la línea de investigación en psicología social y cognitiva que se han centrado en analizar los modos en que las memorias colectivas se forman y transmiten en conversaciones. Luego, relacionamos tales procesos colaborativos con la creación de redes cognitivas distribuidas. Estas generan las condiciones para que podamos aprender, recordar, transmitir y acumular conocimientos, memorias y habilidades a nivel ontogenético. 


\section{Bietti - Construcción de identidades colectivas}

\section{La Formación de la Memoria Colectiva}

Si nos distanciamos un momento del debate filosófico acerca de si los grupos pueden tener una mente y por consiguiente ser capaces de formar memorias colectivas (Bietti, 2012; Sutton, 2008; Theiner, 2013; Wilson, 2005), en términos prácticos podemos definir a la memoria colectiva como memorias individuales compartidas en una comunidad que le confieren identidad a esa comunidad (Coman, Brown, Koppel, \& Hirst, 2009). Las memorias colectivas pueden pertenecer a un proyecto de identidad compartido empleándose para conservar la historia del grupo y mantener su cohesión a través del tiempo (Wertsch, 2002). Varios estudios en psicología social y cognitiva han investigado la influencia de factores sociales, cognitivos y linguiísticos en la formación de memorias colectivas en conversaciones. Estos factores incluyen roles conversacionales (Hirst \& Manier, 1996), la experiencia (Hirst \& Manier, 2008), y el silencio conversacional (Stone, Coman, Brown, Koppel \& Hirst, 2012). Estos estudios demostraron que las formas en las que los miembros de una comunidad comparten sus memorias desempeñan un papel central en la formación y transmisión de las memorias colectivas (Coman et al., 2009; Hirst \& Manier, 2008; Hirst \& Echterhoff, 2012; Muller \& Hirst, 2014).

Los estudios que se centraron en explorar los roles conversacionales en la formación de memorias colectivas en grupos pequeños han identificado tres funciones sociales complementarias que pueden adoptar los participantes: narradores, mentores y supervisores (Hirst \& Manier, 1996; Hirst, Manier \& Apetroaia, 1997). Los participantes que tomaron la iniciativa en las conversaciones sobre experiencias pasadas asumieron el rol de narradores. Estos no solamente hablaron de las experiencias pasadas compartidas con los otros miembros del grupo sino también de experiencias que no fueron compartidas. Luego, los participantes que apoyaron a los narradores proporcionándoles detalles e información adicional para mejorar sus narraciones actuaron como mentores. Y, por último, los que asumieron el rol de supervisores cumplieron la tarea de monitorear y evaluar si las narraciones eran correctas y si no le fueran se encargaban de marcar sus inexactitudes (Hirst \& Manier, 1996). Los tres roles que los participantes en las conversaciones acerca de un pasado compartido pueden asumir son 
flexibles y dinámicos en gran medida y también dependen de las identidades de los interlocutores, de sus objetivos y propósitos específicos (Bietti, 2010).

Otros estudios (ej. Cuc, Ozuru, Manier \& Hirst, 2006) han llevado esta línea de investigación a condiciones más controladas en el laboratorio. Cuc y sus colegas encontraron que en los grupos en los que los interlocutores asumieron el rol de narradores dominantes hubo mayores probabilidades de que se formaran memorias colectivas. Brown, Coman y Hirst (2009) también estudiaron si existían diferencias importantes entre ser un narrador dominante y un experto para la formación de una memoria colectiva. Los autores encontraron que ambas características pueden ser independientes entre sí, es decir, ser un experto no llevó necesariamente a ser un narrador dominante. No obstante, ambas características (ser experto y ser narrador dominante) facilitaron la formación de una memoria colectiva. En relación a los modos en que el silencio conversacional afecta la formación de una memoria colectiva en conversaciones, Stone y sus colegas (Stone et al., 2012) han demostrado que el silencio selectivo acerca de un tópico no sólo induce a que estos tópicos sean menos accesibles, es decir, al olvido, sino que también proporciona un mecanismo funcional para crear una memoria colectiva.

Un estudio reciente investigó cómo el rol de la colaboración influía en la formación de memorias colectivas (Barber, Rajaram, \& Fox, 2012). Barber y sus colegas observaron que incluso cuando la colaboración era negativa para el rendimiento individual en tareas de memoria, ésta fue lo que fomentó la creación de memorias colectivas. Es decir, los autores no encontraron una correlación positiva entre rendimiento individual y la formación de memorias colectivas. Otros estudios han empleado el modelado basado en agentes (MBA) para investigar la transmisión y la propagación de memorias colectivas en grupos sociales artificiales con mayor número de participantes (Luhmann \& Rajaram, 2015). Luhmann y Rajaram (2015) usaron MBA para investigar los efectos de la inhibición de la memoria individual a causa de la colaboración en grupos sociales artificiales de mayor tamaño y complejidad que los analizados anteriormente en el laboratorio. Los autores encontraron efectos similares. Los resultados obtenidos por medio de simulaciones computacionales estuvieron en consonancia con algunos efectos observados en el laboratorio: a medida que los grupos incrementan su número de miembros, se hacen más complejos y se complejiza su colaboración, y 


\section{Bietti - Construcción de identidades colectivas}

aunque la inhibición de la memoria a nivel individual aumenta, también se incrementan significativamente las posibilidades de formar memorias colectivas. Estos resultados están de acuerdo con los hallazgos obtenidos en los estudios que analizaron la transmisión de memorias en cadenas de comunicación (Tan \& Fay, 2011) y las ventajas evolutivas y culturales del contagio social de los recuerdos (ej. Wheeler, 2013). En su conjunto, esta evidencia sugiere que cuestiones de rendimiento en tareas de memoria, inhibición individual de la memoria a causa de la colaboración, el contagio social y la veracidad de las memorias, parecen ser cuestiones bastante independientes cuando se tienen en cuentan los beneficios sociales y evolutivos de recordar en compañía de otras personas (ej. cooperación y la posibilidad de formar una memoria colectiva). Werstch (2009) sostiene que la memoria colectiva se compone de representaciones del pasado distribuidas entre individuos y artefactos culturales. La interacción entre individuos y artefactos culturales ocurre dentro de contextos sociales, históricos y tecnológicos específicos. Es decir, la formación y la transmisión de las memorias colectivas se ve condicionada por recursos biológicos, socio-culturales y tecnológicos así como por las prácticas culturales donde estos elementos interactúan. Un contexto social puede suministrar prácticas culturales que se distinguen de otras en lo que respecta a cómo y qué recordar (Wang, 2013). Esto nos lleva a considerar los modos en que las redes cognitivas distribuidas constituyen contextos enriquecidos para recordar, solos o en compañía de otros, eventos relevantes formadores de identidades individuales y colectivas.

\section{Redes Cognitivas Distribuidas}

Varios autores (Donald, 1991, 1993; Malafouris, 2013; Sterelny, 2012) afirman que el comportamiento humano inteligente tiene sus raíces en interacciones entre las personas, con sus cuerpos adaptables y cerebros plásticos, y los contextos sociales y materiales donde habitan, es decir, no solamente en modelos abstractos o representaciones de conducta humana en nuestros cerebros. La creación de redes cognitivas distribuidas en nuestra historia evolutiva nos permitió aprender, recordar, transmitir y acumular conocimientos y habilidades a lo largo del tiempo. Las redes cognitivas 
distribuidas sirven de andamiaje para construcción de la memoria colectiva en el ámbito de la comunidad. Estas se encuentran conformadas por interfaces entre recursos cognitivos internos y externos. Recursos cognitivos internos incluyen: i) planes motores; ii) respuestas automáticas a un estimulo o señal que hemos aprendido en experiencias pasadas; iii) familiaridad en la forma de exposición repetida a un estímulo perceptual; iv) memorias semánticas (conocimientos generales del mundo); y v) memorias episódicas (recuerdos concretos y detallados) de experiencias que hemos vivido. Por otro lado, recursos cognitivos externos incluyen: i) nuestros contextos sociales y culturales; ii) dispositivos mnemotécnicos; iii) instrumentos de medición; iv) registros escritos (ej. libros); v) notaciones matemáticas; vi) bibliotecas y archivos; así como vii) medios de comunicación electrónicos. Donald (1993) mantiene que, debido a la plasticidad del cerebro, la interacción entre recursos cognitivos internos y externos llevó a continuas adaptaciones de nuestro aparato neuronal. Un ejemplo de tales adaptaciones se puede encontrar en las formas en que la memoria se encuentra adaptando al uso de Internet (ej. Google), en el sentido de que hoy en día solemos recordar mejor donde encontrar la información que ya aprendimos más que la información misma (Sparrow et al., 2011).

Malafouris (2013) reconoce el papel central de la cultura material en la evolución y desarrollo de la cognición humana. Este autor sostiene que el cerebro puede ser concebido como una entidad biológica y un artefacto cultural al mismo tiempo, debido a su continua re-configuración, re-cableado y re-modelado como consecuencia de su interacción con recursos cognitivos externos. Esto nos lleva a pensar que tal vez el costo de tener memorias adaptables y maleables, justamente en lo que reside la 'imperfección' de la memoria humana, pudo haber sido el precio que tuvimos que pagar por su alta capacidad de adaptación a nuestros cambiantes ecosistemas culturales (Hutchins, 2014).

\section{Conclusión}

En este artículo discutimos los resultados encontrados en numerosos estudios, muchos de ellos experimentales, en psicología social, cognitiva y cultural que han investigado los modos en que la memoria humana se adapta 


\section{Bietti - Construcción de identidades colectivas}

a contextos materiales, grupos sociales y las comunidades de las que somos parte. La memoria es uno de los recursos más importantes sosteniendo nuestras historias, acciones y experiencias presentes. Esto la convierte en un ingrediente fundamental de la identidad. La mayoría de estos estudios se han centrado en la vulnerabilidad de nuestras memorias, las cuales se ha comprobado pueden ser fácilmente 'contaminables' con información falsa. Recientemente nuevas líneas de investigación se focalizaron en analizar los beneficios sociales, culturales y evolutivos de contar con memorias adaptables. La adaptabilidad y maleabilidad de la memoria humana es una de las claves para construir y ser parte de grupos sociales. Comunicar nuestras experiencias pasadas es uno de los modos más generalizados de crear nuevas relaciones humanas, fortalecer nuestros grupos de pertenencia, aprender y transmitir nuevos conocimientos y habilidades, $\mathrm{y}$ en última instancia afianzar la confianza. La comunicación y transmisión de nuestras memorias raramente ocurre en el vacío. Ocurre en redes y ecosistemas de naturaleza híbrida que incluyen elementos culturales, sociales y cognitivos. Todo esto crea las condiciones para el surgimiento de memorias colectivas, permitiéndonos así construir narrativas de grupo que nos diferencien de otros grupos. La adaptabilidad y maleabilidad de nuestras memorias, lo que justamente las hace imperfectas y fácilmente 'contaminables', es uno de los factores que hacen que esto pueda ocurrir.

\section{Agradecimientos}

Agradecemos el apoyo del Fonds Nationale de la Recherche Suisse (Subsidio Ambizone P200P1-154968).

\section{Referencias}

Bangerter, A. (2000). Identifying individual and collective acts of remembering in task- related communication. Discourse Processes, 30, 237-264. Doi: https://doi.org/10.1207/S15326950dp3003_2 
Barber, S. J., Rajaram, S., \& Aron, A. (2010). When two is too many: Collaborative encoding impairs memory. Memory \& Cognition, 38, 255264. Doi: https://doi.org/10.3758/MC.38.3.255

Barber, S. J., \& Rajaram, S. (2011). Collaborative memory and part-set cueing impairments: The role of executive depletion in modulating retrieval disruption. Memory, 19, 378-397. Doi: 10.1080/09658211.2011.575787

Barber, S. J., Rajaram, S., \& Fox, E. B. (2012). Learning and remembering with others: The key role of retrieval in shaping group recall and collective memory. Social Cognition, 30, 121-132. Doi:

10.1521/soco.2012.30.1.121

Barnier, A.J., Sutton, J., Harris, C.B., \& Wilson, R.A, (2008). A conceptual and empirical framework for the social distribution of cognition: The case of memory. Cognitive Systems Research, 9(1-2), 33-51. http://dx.doi.org/10.1016/j.cogsys.2007.07.002

Bartlett, F. (1932). Remembering. Cambridge: Cambridge University Press. Basden, B. H., Basden, D. R., \& Henry, S. (2001). Costs and benefits of collaborative remembering. Applied Cognitive Psychology, 14, 497-507. https://doi.org/10.1002/1099-0720(200011/12)14:6<497::AIDACP665>3.0.CO;2-4

Bergman, E., \& Roediger, H. L. (1999). Can Bartlett's repeated reproduction experiments be replicated? Memory \& Cognition, 27, 937-947.

Bietti,L.M. (2010). Sharing memories, family conversation and interaction. Discourse \& Society, 21 (5), 499-523. https://doi.org/10.1177/0957926510373973

Bietti, L. M. (2012). Towards a cognitive pragmatics of collective remembering. Pragmatics \& Cognition, 20(1), 32-61. Doi: 10.1075/pc.20.1.02bie

Bietti, L.M. (2014). Discursive Remembering: Individual and Collective Remembering as a Discursive, Cognitive and Historical Process. Berlin: Walter de Gruyter.

Bietti L.M \& Sutton, J. (2015). Interacting to remember at multiple timescales: Coordination, collaboration, cooperation and culture in joint remembering. Interaction Studies, 16(3), 419-450. Doi:

10.1075/is.16.3.04bie 


\section{Bietti - Construcción de identidades colectivas}

Bietti, L.M, Bangerter, A., Mayor, E. (2017). The interactive shaping of social learning in transmission chains. In G. Gunzelmann, A. Howes, T. Tenbrink. \& E. Davelaar (Eds.), Proceedings of the $39^{\text {th }}$ Annual Conference of the Cognitive Science Society. Austin, TX: Cognitive Science Society, pp. 1641-1646.

Blumen, H., \& Rajaram, S. (2008). Effects of group collaboration and repeated retrieval on individual recall. Memory, 16, 231-244.

Brennan, S. E., Galati, A., \& Kuhlen, A. (2010). Two minds, one dialog: Coordinating speaking and understanding. In B. Ross (Ed.), Psychology of Learning and Motivation, vol. 53. (pp. 301-345). Academic Press/Elsevier.

Brown, A., Coman, A. \& Hirst, W. (2009). Expertise and the formation of collective memory. Social Psychology, 40, 118-128.

Cienki, A., Bietti, L.M., \& Kok, K. (2014). Multimodal alignment during collaborative remembering. Memory Studies, 7(3), 354-369. https://doi.org/10.1177/1750698014530624

Clark, H. H. (1996). Using Language. Cambridge: Cambridge University Press

Clark, H. H., \& Brennan, S. A. (1991). Grounding in communication. In L.B. Resnick, J.M. Levine, \& S.D. Teasley (Eds.). Perspectives on Socially Shared Cognition (pp.127-148). Washington: APA Books.

Coman, A., Brown, A.D., Koppel, J., \& Hirst, W. (2009). Collective memory from a psychological perspective. International Journal of Politics, Culture and Society, 22(2), 125-141. Doi: 10.1007/s10767-0099057-9

Congleton, A. R., \& Rajaram, S. (2011). The influence of learning methods on collaboration: Prior repeated retrieval enhances retrieval organization, abolishes collaborative inhibition, and promotes post-collaborative memory. Journal of Experimental Psychology: General, 140, 535-551. Doi: $10.1037 / \mathrm{a} 0024308$

Cuc, A., Ozuru, Y., Manier, D., \& Hirst, W. (2006). The transformation of collective memories: studies of family recounting. Memory \& Cognition, $34,752-762$.

Cuc, A., Koppel, J., \& Hirst, W. (2007). Silence is not golden: a case for socially-shared retrieval-induced forgetting. Psychological Science, 18, 727-737. Doi: 10.1111/j.1467-9280.2007.01967.x 
Dixon, R.A. (2011) Evaluating everyday competence in older adult couples: epidemiological considerations. Gerontology, 57, 173-179. https://doi.org/10.1159/000320325

Dixon, R.A. (2013). Collaborative memory research in aging: Perspectives on application. Journal of Applied Research in Memory and Cognition, 2(2), 128-130. http://dx.doi.org/10.1016/j.jarmac.2013.05.001

Donald, M. (1991). Origins of the Modern Mind: Three Stages in the Evolution of Culture and Cognition. Cambridge, MA: Harvard University Press. https://doi.org/10.1002/ana.410320432

Donald, M. (1993). Précis of Origins of the modern mind. Behavioral and Brain Sciences, 16, 737-791.

https://doi.org/10.1017/S0140525X00032647

Echterhoff, G., Groll, S., \& Hirst, W. (2007). Tainted truth: Overcorrection for misinformation influence on eyewitness memory. Social Cognition, 25, 367-409. https://doi.org/10.1521/soco.2007.25.3.367

Fagin, M.M., Yamashiro, J.K. \& Hirst, W. (2013). The adaptive function of distributed remembering: Contributions to the formation of collective memory. Review of Philosophy and Psychology, 4(1), 91-106. Doi: 10.1007/s13164-012-0127-y

Gauld, A., \& Stephenson, G.M. (1967). Some experiments related to Bartlett's theory of remembering. British Journal of Psychology, 58, 3949. http://dx.doi.org/10.1111/j.2044-8295.1967.tb01054.x

Harris, C.B., Paterson, H.M. \& Kemp, R.I. (2008). Collaborative recall and collective memory: what happens when we remember together? Memory, 16, 213-230. Doi: 10.1080/09658210701811862

Harris, C.B., Keil, P.G., Sutton, J., Barnier, A., \& McIlwain, D. (2011). We remember, we forget: Collaborative remembering in older couples. Discourse Processes, 48, 267-303. Doi: http://dx.doi.org/10.1080/0163853X.2010.541854

Harris, C.B., Barnier, A.J., \& Sutton, J. (2012). Consensus collaboration enhances group and individual recall accuracy. Quarterly Journal of Experimental Psychology, 65, 179-194. Doi: 10.1080/17470218.2011.608590

Harris, C.B., Barnier, A.J., \& Sutton, J. (2013). Shared encoding and the costs and benefits of collaborative recall. Journal of Experimental 
146 Bietti - Construcción de identidades colectivas

Psychology: Learning, Memory, and Cognition, 39, 183-195. Doi:

10.1037/a0028906

Hirst, W., \& Manier, D. (1996). Social influences on remembering. In D.

Rubin (Ed.), Remembering The Past (pp.271-290). New York:

Cambridge University Press.

Hirst, W., Manier, D., \& Apetroaia, I. (1997). The social construction of the remembered self: Family recounting. Annals of the New York Academy of Sciences, 818, 163-188.

Hirst, W. \& Manier, D. (2008). Towards a psychology of collective memory. Memory, 16(3), 183-20.

https://doi.org/10.1080/09658210701811912

Hirst, W. \& Echterhoff, G. (2012). Remembering in conversations: The social sharing and reshaping of memories. Annual Review of Psychology, 63, 55-69. Doi: 10.1146/annurev-psych-120710-100340

Hollingshead, A.B. (1998). Communication, learning and retrieval in transactive memory systems. Journal of Experimental Social Psychology, 34, 423-442. https://doi.org/10.1006/jesp.1998.1358

Hutchins, E. (2014). The cultural ecosystem of human cognition.

Philosophical Psychology, 27 (1), 34-49.

https://doi.org/10.1080/09515089.2013.830548

Hyman, I. E., Cardwell, B. A., \& Roy, R. A. (2013). Multiple causes of collaborative inhibition in memory for categorised word lists. Memory, 21(7), 875-890. Doi: 10.1080/09658211.2013.769058

Jackson, M., \& Moreland, R.L. (2009). Transactive memory in the classroom. Small Group Research, 40(5), 508-534. Doi: https://doi.org/10.1177/1046496409340703

Kashima, Y. (2000). Maintaining cultural stereotypes in the serial reproduction of narratives. Personality and Social Psychology Bulletin, 26(5), 594-604. Doi: https://doi.org/10.1177/0146167200267007

Lewis, K., Lange, D., \& Gillis, L. (2005). Transactive memory systems, learning, and learning transfer. Organization Science, 16(6), 581-598. Doi: $10.1287 /$ orsc. 1050.0143

Loftus, E.F. (1979). The malleability of human memory. American Scientist, $67,312-320$. 
Loftus, E.F. (2005). Planting misinformation in the human mind: a 30-year investigation of the malleability of memory. Learning and Memory, 12(4), 361-366. Doi: 10.1101/1m.94705.

Luhmann, C.C. \& Rajaram, S. (2015). Memory transmission in small groups and large networks: An agent based model. Psychological Science, 26, 1909-1917. Doi: 10.1177/0956797615605798

Malafouris, L (2013). How Things Shape the Mind: A Theory of Material Engagement. Cambridge, MA: MIT Press.

https://doi.org/10.1111/etho.12074

Meade, M. L., \& Roediger, H. L., III. (2002). Explorations in the social contagion of memory. Memory \& Cognition, 30, 995-1009. https://doi.org/10.3758/BF03194318

Meade, M.L., Nokes, T.J. \& Morrow, D.G. (2009). Expertise promotes facilitation on a collaborative memory task. Memory, 17, 39-48. Doi: 10.1080/09658210802524240

Mesoudi, A. \& Whiten, A. (2004). The hierarchical transformation of event knowledge in human cultural transmission. Journal of Cognition and Culture, 4(1), 1-24. Doi: 10.1163/156853704323074732

Mesoudi, A. \& Whiten, A. (2008). The multiple roles of cultural transmission experiments in understanding human cultural evolution.

Philosophical Transactions of the Royal Society B, 363, 3489-3501. Doi: 10.1098/rstb.2008.0129

Michaelian. K. \& Sutton, J. (2013). Distributed cognition and memory research: History and current directions. Review of Philosophy and Psychology, 4(1), 1-24. Doi: 10.1007/s13164-013-0131-X

Middleton, D. \& Brown, S.D. (2005) The Social Psychology of Experience:

Studies in Remembering and Forgetting. London: Sage.

http://dx.doi.org/10.4135/9781446221808

Muller, F. \& Hirst, W. (2014). Remembering stories together: Social contagion and the moderating influence of disagreements in conversations. Journal or Applied Research in Memory and Cognition, 3, 7-11. http://dx.doi.org/10.1016/j.jarmac.2013.12.002

Numbers, K.T., Meade, M.L., \& Perga, V.A. (2014). The influences of partner accuracy and partner memory ability on social false memories. Memory \& Cognition. doi:10.3758/s13421-014-0443-9. http://dx.doi.org/10.3758/s13421-014-0443-9 


\section{Bietti - Construcción de identidades colectivas}

Pereira-Pasarin, L. \& Rajaram, S. (2011). Study repetition and divided attention: Effects of encoding manipulations on collaborative inhibition in group recall. Memory \& Cognition, 39, 968-97.

https://doi.org/10.3758/s13421-011-0087-y

Rajaram, S. (2011). Collaboration both hurts and helps memory: A cognitive perspective. Current Directions in Psychological Science, 20, 76-81. Doi: 10.1177/0963721411403251

Rajaram, S. \& Pereira-Pasarin, L.P. (2010). Collaborative memory:

Cognitive research and theory. Perspectives on Psychological Science, 5, 649-663. https://doi.org/10.1177/1745691610388763

Ren, Y. \& Argote, A. (2011). Transactive memory systems 1985-2010:

An integrative framework of key dimensions, antecedents and consequences. The Academy of Management Annals, 5(1), 189-229. https://doi.org/10.1080/19416520.2011.590300

Roediger, H. L. III, Meade, M. L., \& Bergman, E. (2001). Social contagion of memory. Psychonomic Bulletin \& Review, 8, 365-371. https://doi.org/10.3758/BF03196174

Roediger H. L. III, Meade, M. L., Gallo, D. A., \& Olson, K. R. (2014).

Bartlett revisited: Direct comparison of repeated reproduction and serial reproduction techniques. Journal of Applied Research in Memory and Cognition. Doi: 10.1016/j.jarmac.2014.05.004.

Sparrow, B., Liu, J., \& Wegner, D. M. (2011). Google effects on memory:

Cognitive consequences of having information at our fingertips. Science, 333, 776-778. Doi: 10.1126/science. 1207745

Sterelny, K. (2012). The Evolved Apprentice: How Evolution Made Humans Unique. Cambridge, MA: MIT Press.

Stone, C., Coman, A., Brown, A.D., Koppel, J., \& Hirst, W. (2012). Toward a science of silence: The consequences of leaving a memory unsaid.

Perspectives in Psychological Science, 7 (1), 39-53.

https://doi.org/10.1177/1745691611427303

Suddendorf, T. \& Corballis, M.C. (2007). The evolution of foresight: What is mental time travel and is it unique to humans? Behavioral and Brain Sciences, 30, 299-313. Doi: 10.1017/S0140525X07001975

Sutton, J. (2008). Between Individual and Collective Memory: interaction, coordination, distribution. Social Research, 75(1), 23-48. 
Sutton J., Harris, C.B., Keil, P.G., \& Barnier, A. J. (2010). The psychology of memory, extended cognition, and socially distributed remembering. Phenomenology and the Cognitive Sciences, 9, 521-560. Doi:

10.1007/s11097-010-9182-y

Tan, R., \& Fay, N. (2011). Cultural transmission in the laboratory: Agent interaction improves the intergenerational transfer of information. Evolution \& Human Behavior, 32(6), 399-406.

Theiner G. (2013). Transactive memory systems: A mechanistic analysis of emergent group memory. Review of Philosophy and Psychology, 4(1), 65-89. Doi: 10.1007/s13164-012-0128-x

Wagoner, B \& Gillespie, A. (2013). Sociocultural mediators of remembering: An extension of Bartlett's method of repeated reproduction. British Journal of Social Psychology, doi:

10.1111/bjso.12059. Doi: 10.1111/bjso.12059

Wang, Q. (2013). The Autobiographical Self in Time and Culture. New York: Oxford University Press. Doi:

http://dx.doi.org/10.1093/acprof:oso/9780199737833.001.0001

Wegner, D.M. (1986). Transactive memory: A contemporary analysis of the group mind. In B. Mullen \& G.R. Goethals (Eds.), Theories of Group Behavior (pp.185-208). New York: Springer.

Wegner, D. M., Erber, R., \& Raymond, P. (1991). Transactive memory in close relationships. Journal of Personality and Social Psychology, 61, 923-929.Doi: http://dx.doi.org/10.1037/0022-3514.61.6.923

Weldon, M. S., \& Bellinger, K. D. (1997). Collective memory: Collaborative and individual processes in remembering. Journal of Experimental Psychology: Learning, Memory, and Cognition, 23, 11601175. Doi: http://dx.doi.org/10.1037/0278-7393.23.5.1160

Wertsch, J.V. (2002). Voices of Collective Remembering. Cambridge:

Cambridge. University Press.

Wertsch, J. V (2009). Collective memory. In P. Boyer \& J.V. Wertsch (Eds.), Memory in Mind and Culture (pp. 117-137). Cambridge:

Cambridge University Press. Recuperado de https://doi.org/10.1017/CBO9780511626999.008

Wheeler, M. A., \& Roediger III, H. L. (1992). Disparate effects of repeated testing: Reconciling Ballard's (1913) and Bartlett's (1932) results. 


\section{Bietti - Construcción de identidades colectivas}

Psychological Science, 3, 240-24. Doi: https://doi.org/10.1111/j.14679280.1992.tb00036.x

Wheeler, R., Allan, K., Tsivilis, D., Martin, D. \& Gabbert, F. (2013).

Explicit mentalizing mechanisms and their adaptive role in memory conformity. PLoS ONE 48 8(4): e62106. Doi:

10.1371/journal.pone.0062106.

Wilson, R. A. (2005). Collective memory, group minds, and the extended mind thesis. Cognitive Processing, 6, 227-236. Doi: 10.1007/s10339005-0012-z

Xu, J. \& Griffiths, T. L. (2010). A rational analysis of the effects of memory biases on serial reproduction. Cognitive Psychology, 60(2), 107-126.

Doi: 10.1016/j.cogpsych.2009.09.002

Zerubavel, E. (2003). Time Maps: Collective Memory and the Social Shape of the Past. Chicago: University of Chicago Press.

Lucas Bietti: Investigador de la Universidad de Neuchâtel, Suiza. http://orcid.org/0000-0002-4380-2615

Contact Address: lucbietti@gmail.com 